\title{
p53-positive expression in dysplastic surgical margins is a predictor of tumor recurrence in patients with early oral squamous cell carcinoma
}

This article was published in the following Dove Medical Press journal: Cancer Management and Research

\author{
Xi-Hu Yang ${ }^{1, *}$ \\ Liang Ding $2, *$ \\ Yong $\mathrm{Fu}^{\prime}$ \\ Sheng Chen ${ }^{3}$ \\ Lei Zhang ${ }^{3}$ \\ Xiao-Xin Zhang ${ }^{2}$ \\ Xiao-Feng Huang ${ }^{3}$ \\ Zhan-Yi Lu' \\ Yan-Hong $\mathrm{Ni}^{2}$ \\ Qin-Gang $\mathrm{Hu}^{\prime}$
}

'Department of Oral and Maxillofacial Surgery, Nanjing Stomatological Hospital, Medical School of Nanjing University, Nanjing, Jiangsu 210000 ,

China; ${ }^{2}$ Central Laboratory of

Stomatology, Nanjing Stomatological

Hospital, Medical School of Nanjing

University, Nanjing, Jiangsu 210000 ,

China; ${ }^{3}$ Department of Oral Pathology,

Nanjing Stomatological Hospital,

Medical School of Nanjing University,

Nanjing, Jiangsu 210000, China

*These authors contributed equally to this work

Correspondence: Yan-Hong Ni Central Laboratory of Stomatology,

Nanjing Stomatological Hospital, Medical School of Nanjing University, No. 30

Zhongzhong Road, Nanjing, Jiangsu

210000, China

Email Niyanhong12@163.com

Qin-Gang Hu

Department of Oral and Maxillofacial Surgery, Nanjing Stomatological Hospital, Medical School of Nanjing University, No. 30 Zhongzhong Road, Nanjing, Jiangsu 210000, China

Email qinganghu@hotmail.com
Purpose: This was a retrospective analysis of the impact of the expression of p53 in the dysplastic surgical margins of early oral squamous cell carcinoma (OSCC) (pT1-2, N0).

Patients and methods: Seventy-two patients with early oral squamous cell carcinoma (OSCC) were recruited. Margin characteristics were abstracted from the pathology report. Expression of p53 in dysplastic surgical margins was examined with the immunohistochemical method and was correlated with clinicopathological parameters and clinical outcomes.

Results: Patients with moderate/severe dysplasia had poor local relapse-free survival (RFS) compared to those with mild dysplasia. Thirty-two (44.4\%) had at least one p53-positive margin, and there was a significant association between the expression of $\mathrm{p} 53$ and tumor recurrence $(P<0.001)$. p53-positive expression was correlated with RFS in patients with dysplastic margins, and its expression in moderate/severe dysplastic groups had a worse RFS than mild dysplastic groups. We also found that the grade of the dysplasia margin was not correlated with RFS in p53-negative groups. Multivariable analysis validated p53 expression in dysplastic surgical margins as an independent risk factor for recurrence.

Conclusion: Our results validated that p53 expression was an independent risk factor for early OSCC with dysplastic surgical margins. Additional therapy and close follow-up are needed for these patients.

Keywords: OSCC, dysplastic surgical margins, p53, oral squamous cell carcinoma, dysplastic surgical margins, prognosis

\section{Introduction}

Only modest improvement has been made in the survival of patients treated for head and neck carcinoma over the last 30 years, in part because obtaining clear resection margins is difficult. Pathology in the margin is an important prognostic factor for relapse-free survival (RFS) in head and neck cancer managed with primary surgery because remaining tumor cells or dysplastic epithelial cells in the margins may lead to the local recurrence (LR) of oral squamous cell carcinoma (OSCC). ${ }^{1-3}$ Even if the histological status of surgical margins is "clear", the LR rate remains between $10 \%$ and 30\%. ${ }^{4}$ Traditionally, surgeons and pathologists have classified surgical margins as involved margins (margin $\leq 1 \mathrm{~mm}$ ), close margins (margin $1-5 \mathrm{~mm}$ ), or clear margins (margin $>5 \mathrm{~mm}){ }^{3}$

While considerable data characterizing the influence of residual carcinoma at the margin are available, the impact of dysplastic margins on prognosis is less clear. Additionally, excising an area of dysplasia adjacent to an invasive cancer in the course 
of an extirpative procedure can affect quality of life, with larger resections generally resulting in a reduced functional outcome. Generally, severe dysplasia (carcinoma in situ) and invasive carcinoma at the margin are considered a "positive margin" needing additional resection, while both moderate and mild dysplasia at the margin do not warrant further surgery. The prognostic importance of severe dysplasia at the margin appears significant, while the prognostic importance of moderate and mild dysplasia at the margin is relatively unclear. Up to now, dedicated reports describing the outcomes of moderate and mild dysplasia are sparse, and management techniques range from re-excision for any dysplasia to close observation and are pursued without the benefit of a marker which can predict the patient's prognosis. ${ }^{5}$

We hypothesized dysplasia margins that share the same changes in marker expression as those observed in OSCC. Therefore, it is important to identify molecular markers that correlate with relapse in patients with dysplastic margins. p53 is a classic tumorsuppressor protein that correlates with the carcinogenesis of OSCC. Brennan et al were the first to validate the potential utility of the $\mathrm{p} 53$ protein as a molecular marker in the assessment of "molecular surgical margins" for patients with OSCC. ${ }^{6}$ Therefore, we performed immunohistochemical analyses to assess changes in the $\mathrm{p} 53$ protein in dysplasia margins to establish whether the marker could predict LR in patients with dysplastic margins.

\section{Patients and methods}

\section{Patients and tumor specimens}

This study was approved by the medical ethics committee of the Stomatological Hospital, Medical School of Nanjing University and was carried out according to the recommendations of the Declaration of Helsinki. Seventy-two patients with early OSCC and who met the following inclusion criteria were recruited: 1) all the patients were diagnosed with pT1-2, N0 OSCC according to the American Joint Committee on Cancer guidelines; 2) all the tumor biopsy specimens were diagnosed as invasive OSCC; 3 ) all the patients had at least an initial frozen-section dysplastic margin: mild 45, moderate 17 , severe $10 ; 4)$ no radiotherapy or chemotherapy was received before or after surgery. All patients were treated with primary surgical resection alone at the Stomatological Hospital, Medical School of Nanjing University between 2007 and 2014. Dysplasia was reported as either mild, moderate, or severe according to the WHO criteria. ${ }^{7}$ Severe/moderate dysplasia at an initial frozen-section margin was additionally excised, while additional resection in a separate surgical procedure was recommended for those with severe/moderate dysplasia at a final margin. During the study period, mild dysplasia at the margin was not re-resected when assigned by the frozen section analysis. LR was defined as tumor regrowth $<2 \mathrm{~cm}$ away from the index tumor and occurring within 3 years. ${ }^{8}$ Smoking was scored as $\mathrm{Y}$ (yes) if a patient had a history of smoking and $\mathrm{N}$ (no) if a patient had never smoked. The follow-up status of the patients was obtained by contacting the patients or their relatives. The mean follow-up time was 57.1 months (range 6-124 months).

\section{Immunohistochemistry}

Immunohistochemistry was used to detect monoclonal mouse anti-p53 (Abcam, Cambridge, UK) in margin sections from all 72 patients according to the manufacturer's protocol, and PBS and normal margin was used as negative controls. Margins that contained $>50$ parabasal or suprabasal cell nuclei in the dysplastic epithelium were considered positive. ${ }^{9}$

\section{Statistical analysis}

The SPSS 17.0 software package was used for statistical analysis. Chi-square tests were used to determine the correlation between $\mathrm{p} 53$ and the clinicopathological data of the patients. We estimated survival curves using the KaplanMeier method and compared them using a two-sided logrank test. The Cox proportional hazards model was used to investigate the predictor for local RFS, and the factors with significant difference in univariate Cox analysis were included in a multivariable Cox regression. $P<0.05$ was considered statistically significant.

\section{Results \\ Clinical characteristics}

Seventy-two patients with early OSCC (pT1-T2, N0) were enrolled in the study. The clinical details of the 72 patients are shown in Table 1 . The study included 31 women and 41 men with a median age of 53.0 years (range $31-77$ ). Of the 72 patients, $29(40.2 \%)$ were tobacco consumers. During the follow-up period, 12 patients $(16.6 \%)$ died, and 16 patients (22.2\%) experienced LR.

\section{Patients with moderate/severe dysplasia had poor RFS}

The relationship between the grade of dysplastic margin, pathologic grade, and smoking history was not significant (Table 1). Tumor recurrence was close to statistical significance $(P=0.072)$. The Kaplan-Meier estimates and log-rank tests showed the grades of dysplastic margin (mild/moderate/ severe) were not correlated with RFS (Figure 1A). As moderate dysplasia and severe dysplasia at the margin appeared to behave in a similar clinical fashion, we divided the patients 
into two groups: a mild dysplasia group and a moderate/ severe dysplasia group. The survival curves validated the conclusion that the moderate/severe dysplasia group had poor RFS (Figure 1B). Our result was consistent with those of previous reports.

\section{p53 expression pattern in dysplastic margins}

Next, we examined the expression of p53 in dysplastic margins. Table 2 shows the immunohistochemistry quanti- fication score. p53 was mainly localized in the basal layer of epithelial cells with some expression in the suprabasal layer (Figures 2 and 3; Table 3), and 32 (44.4\%) had at least one p53-positive margin (Table 4). No significant difference was found between mild, moderate, and severe dysplasia (Figure 4). The expression of p53 at the dysplastic margins was positively correlated with tumor recurrence $(P<0.001)$, but in these patients with early OSCC it was not correlated with pathologic grade, grade of dysplasia, or smoking history (Table 4).

Table I Patient characteristics according to grade of margin dysplasia

\begin{tabular}{|c|c|c|c|c|}
\hline Characteristics & Mild $(n=47)$ & Moderate $(n=17)$ & Severe $(n=8)$ & P-value \\
\hline \multicolumn{5}{|l|}{ Age (years) } \\
\hline$<60$ & $21(44.7 \%)$ & $6(35.3 \%)$ & $4(50 \%)$ & 0.766 \\
\hline$\geq 60$ & $26(55.3 \%)$ & II (64.7\%) & $4(50 \%)$ & \\
\hline \multicolumn{5}{|l|}{ Sex } \\
\hline Male & $26(55.3 \%)$ & $12(70.6 \%)$ & $3(37.5 \%)$ & 0.295 \\
\hline Female & $21(44.7 \%)$ & $5(29.4 \%)$ & $5(62.5 \%)$ & \\
\hline \multicolumn{5}{|l|}{ Smoking history } \\
\hline Yes & $20(42.5 \%)$ & 7 (4I.2\%) & $2(25 \%)$ & 0.714 \\
\hline No & $27(57.5 \%)$ & $10(58.8 \%)$ & $6(75 \%)$ & \\
\hline \multicolumn{5}{|l|}{ Histological grade } \\
\hline Well differentiated & 39 (82.9\%) & $15(88.2 \%)$ & $6(75 \%)$ & 0.792 \\
\hline Poorly differentiated & $8(17.1 \%)$ & $2(11.8 \%)$ & $2(25 \%)$ & \\
\hline \multicolumn{5}{|l|}{ Recurrence } \\
\hline Yes & 7 (14.9\%) & $6(35.3 \%)$ & $3(37.5 \%)$ & 0.072 \\
\hline No & $4(85.1 \%)$ & II (64.7\%) & $5(62.5 \%)$ & \\
\hline
\end{tabular}

A

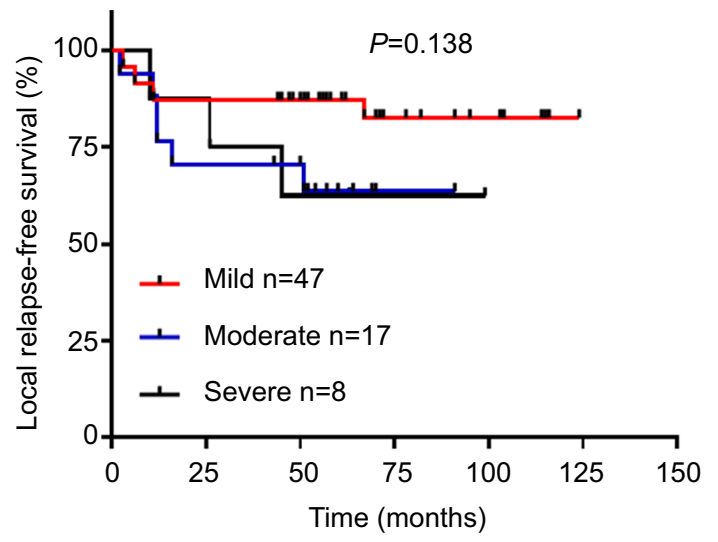

B

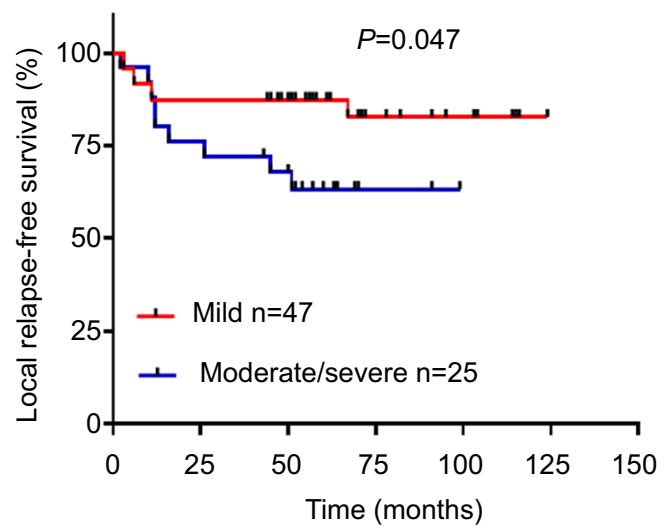

Figure I Grade of dysplastic margin and relapse-free survival.

Notes: (A) Mild vs moderate vs severe. (B) Mild vs moderate/severe.

Table 2 Score table of immunohistochemistry quantification

\begin{tabular}{|c|c|c|c|c|}
\hline Intensity (score) & Number o & (score) & & \\
\hline & $0-50(0)$ & $50-75(1)$ & $75-100(2)$ & $>100(3)$ \\
\hline Weak staining $(\mathrm{I})$ & $0(2 / 2.77 \%)$ & $\mathrm{I}(13 / 18 . \%)$ & $2(4 / 5.5 \%)$ & $3(1 / 1.38 \%)$ \\
\hline Moderate staining (2) & $0(2 / 2.77 \%)$ & $2(2 / 2.77 \%)$ & $4(4 / 5.5 \%)$ & $6(2 / 2.77 \%)$ \\
\hline Strong staining (3) & $0(1 / 1.38 \%)$ & $3(2 / 2.77 \%)$ & $6(2 / 2.77 \%)$ & $9(2 / 2.77 \%)$ \\
\hline
\end{tabular}




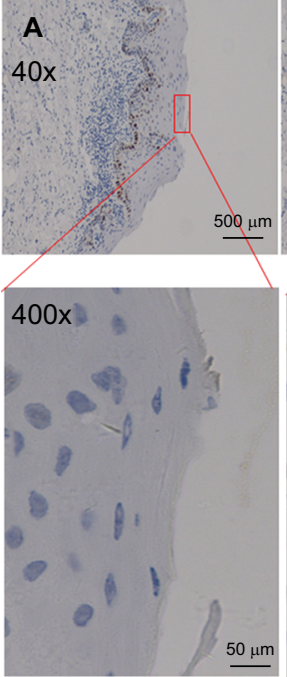

Surface/middle layer
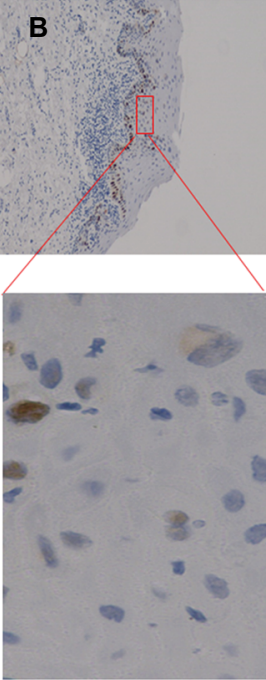

Spinous layer
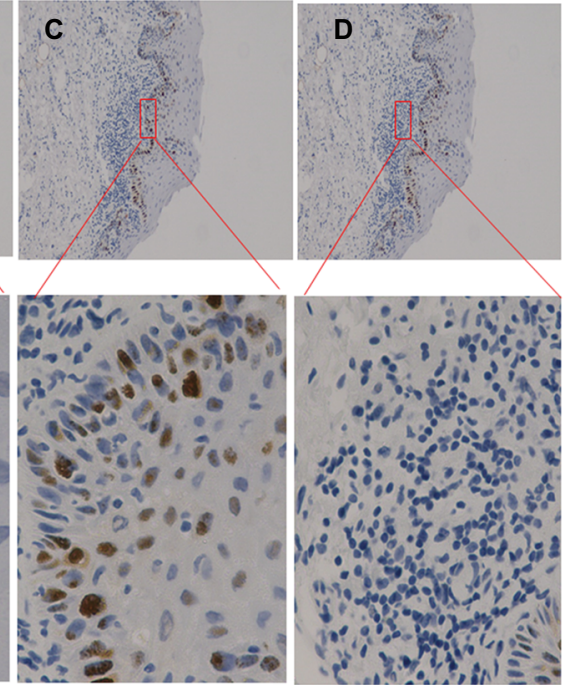

Basal layer

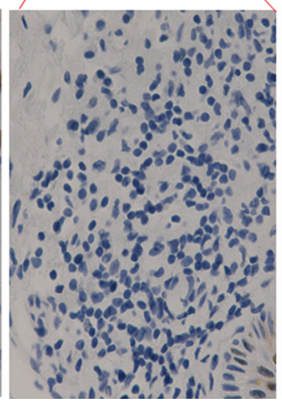

Lamina propria
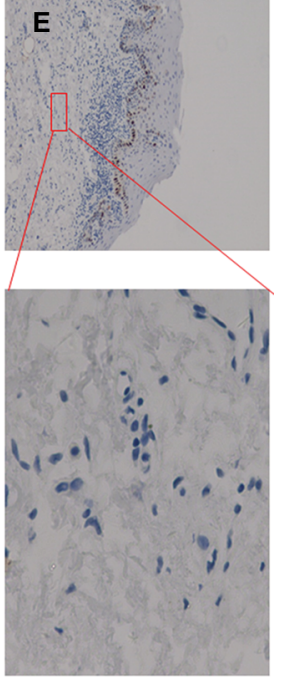

Submucosa

Figure 2 p53 expression pattern in oral epithelial mucosa.

Notes: (A) Surface/middle layer. (B) Spinous layer. (C) Basal layer. (D) Lamina propria. (E) Submucosa. Scale bar: $500 \mu \mathrm{m}(40 \times)$, $50 \mu \mathrm{m}(400 \times)$.
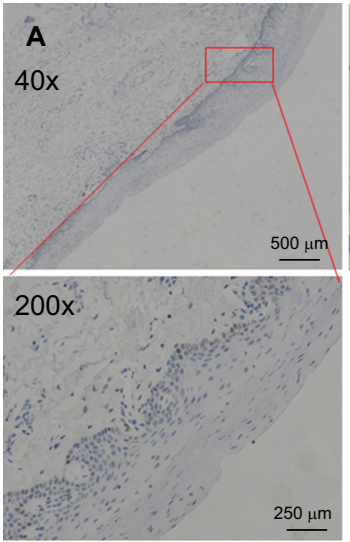

Normal margin

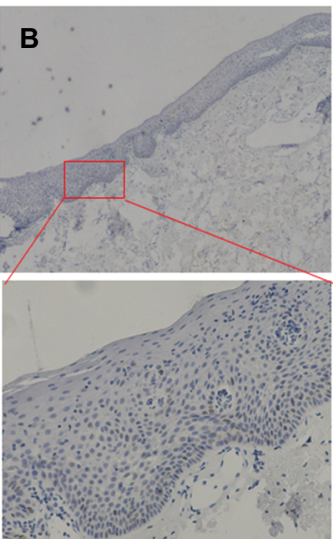

Mild dysplasia

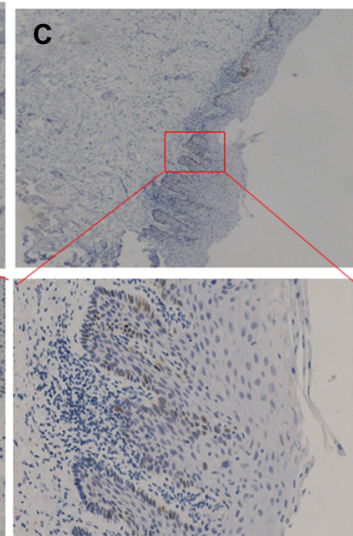

Moderate dysplasia

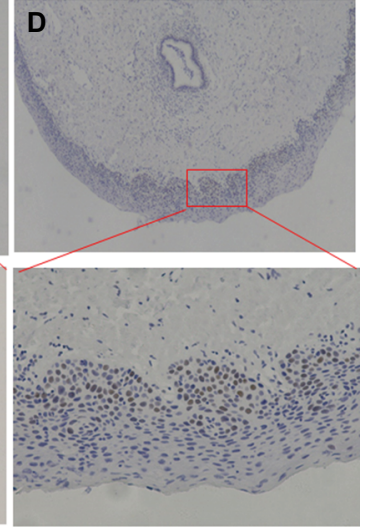

Severe dysplasia

Figure 3 p53 expression in dysplastic margin.

Notes: (A) Normal margin. (B) Mild dysplasia. (C) Moderate dysplasia. (D) Severe dysplasia. Scale bar: $500 \mu \mathrm{m}(40 \times), 250 \mu \mathrm{m}(200 \times)$.

Table 3 Expression of p53 in different locations of oral epithelial mucosa

\begin{tabular}{|l|l|}
\hline Location & Number of p53-positive cells \\
\hline Surface/middle layer & 0 \\
\hline Spinous layer & 3 \\
\hline Basal layer & 29 \\
\hline Lamina propria & 0 \\
\hline Submucosa & 0 \\
\hline
\end{tabular}

\section{p53-positive expression in moderate/ severe dysplastic groups had a worse RFS than mild dysplastic groups}

In order to analyze the effect of p53-positive expression on treatment outcome in different grades of dysplastic margins, we divided the patients into three groups: mild/moderate/severe dysplasia groups. We found that p53-positive expression was more strongly associated with recurrence in the mild and severe dysplasia groups ( $P=0.012$ and $P=0.018$ respectively, Table 5). p53 expression was correlated with RFS in moderate/severe dysplasia groups (Figure 5B, C). Mild dysplasia was not correlated with RFS, but it was close to statistical significance (Figure 5A).

\section{Grade of dysplastic margin was not correlated with RFS in patients with p53- negative expression}

The Kaplan-Meier estimates and log-rank tests showed that p53-positive expression in dysplastic margins was cor- 
Table 4 p53 expression in dysplasia margin

\begin{tabular}{|l|l|l|l|}
\hline \multirow{2}{*}{ Characteristics } & \multicolumn{3}{|l|}{ p53 expression } \\
\cline { 2 - 4 } & $\begin{array}{l}\text { Positive } \\
\text { (n=32) }\end{array}$ & $\begin{array}{l}\text { Negative } \\
\mathbf{( n = 4 0 )}\end{array}$ & P-value \\
\hline Age (years) & & & \\
\hline$<60$ & $12(37.5 \%)$ & $19(47.5 \%)$ & 0.394 \\
\hline$\geq 60$ & $20(62.5 \%)$ & $21(52.5 \%)$ & \\
\hline Sex & & & \\
\hline Male & $16(50 \%)$ & $25(62.5 \%)$ & 0.287 \\
\hline Female & $16(50 \%)$ & $15(37.5 \%)$ & \\
\hline Smoking history & & & \\
\hline Yes & $12(37.5 \%)$ & $17(42.5 \%)$ & 0.667 \\
\hline No & $20(62.5 \%)$ & $23(57.5 \%)$ & \\
\hline Histological grade & & & \\
\hline Well differentiated & $26(81.2 \%)$ & $34(85 \%)$ & 0.671 \\
\hline Poorly differentiated & $6(18.8 \%)$ & $6(15 \%)$ & \\
\hline Recurrence & & & \\
\hline Yes & $14(43.7 \%)$ & $2(5 \%)$ & $<\mathbf{0 . 0 0 I}$ \\
\hline No & $18(56.3 \%)$ & $38(95 \%)$ & \\
\hline Grade of dysplasia & & & \\
\hline Mild & $20(62.5 \%)$ & $27(67.5 \%)$ & 0.697 \\
\hline Moderate & $9(28.1 \%)$ & $8(20 \%)$ & \\
\hline Severe & $3(9.4 \%)$ & $5(12.5 \%)$ & \\
\hline Not Stistcly sifn & & \\
\hline
\end{tabular}

Note: Statistically significant values are shown in bold.

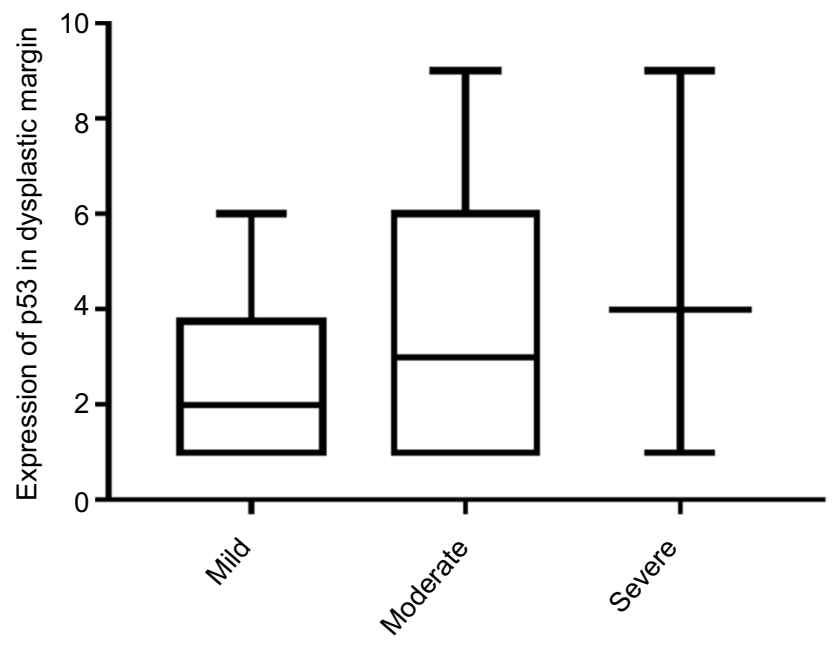

Figure 4 p53 expression score in dysplastic margin.

Table 5 p53 status combine with grade of surgical margin dysplasia in relation to the disease reccurence

\begin{tabular}{|l|l|l|l|}
\hline & \multicolumn{2}{|l|}{ Tumor reccurence } & \multirow{2}{*}{ P-value } \\
\cline { 1 - 3 } P53/grade of dysplasia & Yes & No & \\
\hline P53+/Mild dysplasia & $6(30 \%)$ & $14(70 \%)$ & \multirow{2}{0.012}{} \\
P53-/Mild dysplasia & $\mathrm{I}(3.7 \%)$ & $26(96.3 \%)$ & \\
\hline P53+/Moderate dysplasia & $5(55.6 \%)$ & $4(44.4 \%)$ & $0.13 \mathrm{I}$ \\
P53-/Moderate dysplasia & $\mathrm{I}(12.5 \%)$ & $7(87.5 \%)$ & \\
\hline P53+/Severe dysplasia & $3(100 \%)$ & $0(0 \%)$ & $\mathbf{0 . 0 1 8}$ \\
P53-/Severe dysplasia & $0(0 \%)$ & $5(100 \%)$ & \\
\hline
\end{tabular}

Note: Statistically significant values are shown in bold. related with local RFS (Figure 6A). We further analyzed the relationships between the grade of dysplastic margin and RFS in the p53-positive group and the p53-negative group. The result showed that the grade of dysplastic margin was correlated with RFS in the p53-positive group (Figure 6B), but not in the p53-negative group (Figure 6C). Multivariable analysis validated the hypothesis that $\mathrm{p} 53$ expression in dysplastic margins was an independent risk factor for recurrence (Table 6).

\section{Discussion}

LR after surgery is often indicated by the presence of residual cancer or dysplastic cells in the OSCC surgical margins..$^{10,11}$ However, the relationship between the degree of dysplasia and tumor recurrence has not been fully elucidated, especially with a lack of molecular markers to predict recurrence for OSCC with dysplastic surgical margins. Sopka et $\mathrm{al}^{12}$ reported that severe/moderate dysplasia at the surgical margin correlated with LR for early stage tongue cancer. Weijers et $\mathrm{al}^{13}$ reported that mild/moderate epithelial dysplasia in the margins of OSCC was indicative of a significant risk for LR. Our team ${ }^{14}$ also demonstrated that mild epithelial dysplasia correlated with LR and RFS. p53 is a classic tumor suppressor gene, and its mutation is closely related to the occurrence and development of tumors. It has been used to evaluate molecular surgical margins in OSCC. ${ }^{15}$ p53 mutations in surgical margins (deep or mucosa margin) have been shown to be valuable in predicting LR in patients with head and neck squamous cell carcinoma, even with negative margins. ${ }^{16-21}$ Because the detection of markers in the dysplastic surgical margins can easily be performed within 2 weeks, p53 may be a useful marker to make postoperative decisions for patients with OSCC and dysplastic surgical margins. ${ }^{21}$ In most patients with OSCC, the recurrence after surgery was a result of genetically changed fields around the primary tumor. ${ }^{22,23}$

The pathologic evaluation of oral epithelial dysplasia is dependent upon the thickness of the involved epithelium and is subject to significant intra- and inter-observer differences in the grading of specimens. Severe dysplasia and carcinoma in situ have been considered positive margins that need further surgical excision..$^{24}$ As a uniform standard is lacking for further resection of mild and moderate dysplasia, molecular markers are urgently needed to evaluate dysplastic margins, especially for mild and moderate dysplasia.

In the present study, the relationship between the grades of dysplastic margins and tumor recurrence was not significant, but did approach statistical significance. When we divided the patients into mild dysplasia and moderate/ 
A
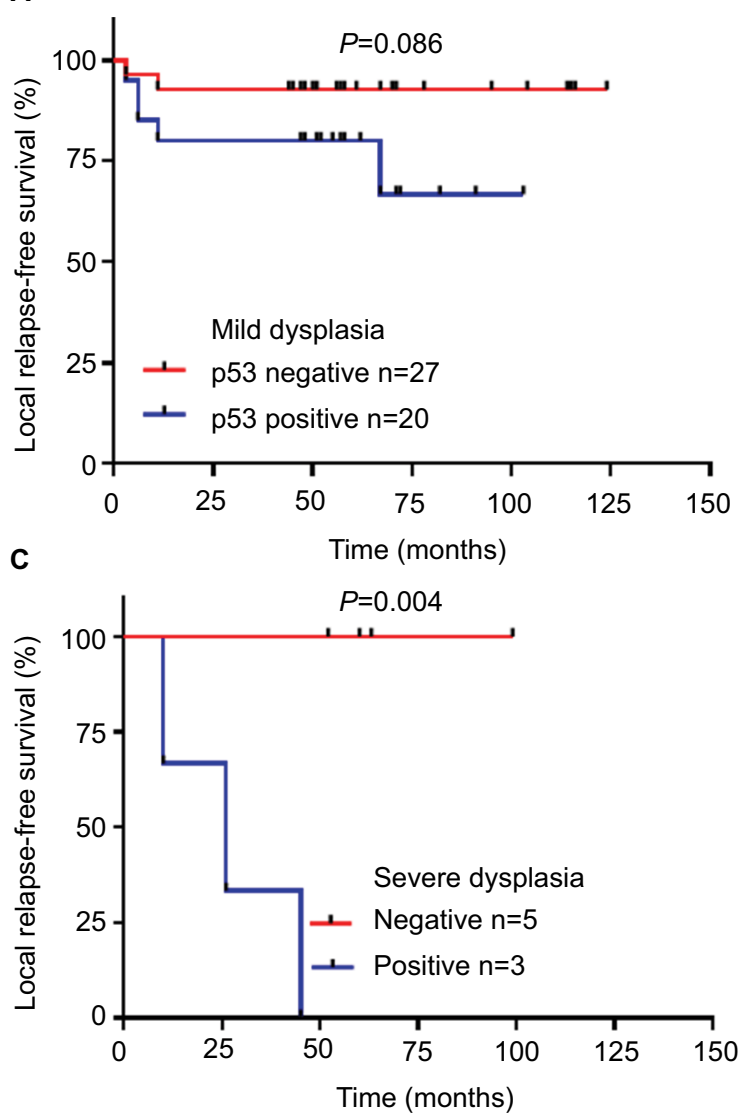

B

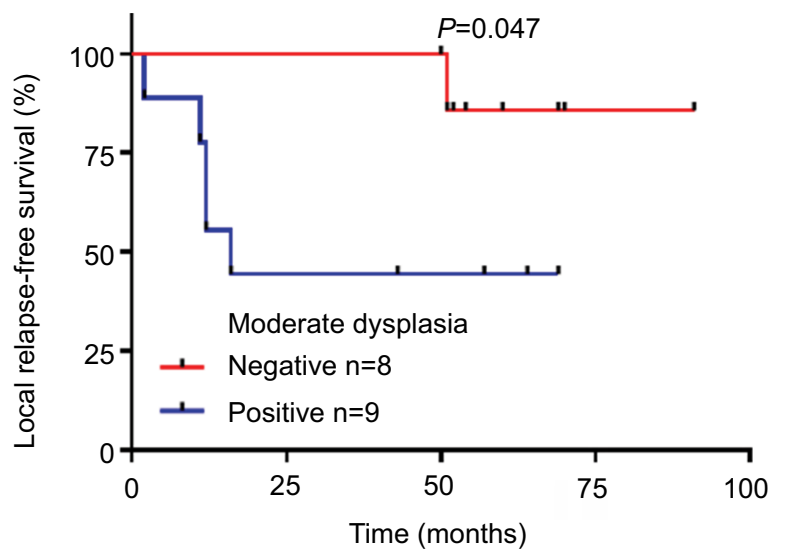

Figure 5 Relationship between p53 expression and relapse-free survival in mild/moderate/severe dysplasia groups. Notes: (A) Mild. (B) Moderate. (C) Severe.

severe dysplasia groups, the recurrence rate in the moderate/ severe group $(9 / 25,36 \%)$ was significantly higher than in the mild group $(7 / 47,14.9 \%)$ and had poorer RFS, results that are consistent with a previous report. ${ }^{12}$ For $\mathrm{p} 53$ expression in patients with dysplastic surgical margins, a significant association was found between the expression of $\mathrm{p} 53$ in the dysplastic margins and tumor recurrence, indicating a poor RFS. An analysis of the effect of p53 expression status on RFS with different grades of dysplastic margins showed that the grade of dysplastic margin was correlated with RFS in the p53-positive group, but not in the p53-negative group. These data suggest that p53 expression may contribute to tumor recurrence. Multivariate analysis also validated the hypothesis that $\mathrm{p} 53$ expression in dysplastic surgical margins was an independent prognostic factor for LR in patients with early OSCC.

We further analyzed the effect of p53-positive expression on treatment outcomes with different grades of dysplastic margin. We found that $\mathrm{p} 53$-positive expression was more strongly associated with recurrence in the mild and severe dysplasia groups ( $P=0.012$ and $P=0.018$, respectively). p53 expression was correlated with RFS in the moderate/severe dysplasia group (Figure 5). Mild dysplasia was not correlated with RFS, but was close to statistical significance. Therefore, close follow-up is recommended for patients with p53-positive expression.

This study was limited in that it was a retrospective analysis with a small sample size. We chose immunohistochemistry to evaluate p53 expression instead of a quantitative method primarily because fresh biopsy tissues were not available. Although immunohistochemistry is a semi-quantitative method, it is currently the most commonly used, the most straightforward, and the most costeffective protocol. ${ }^{25}$ Many factors likely contribute to the malignant transformation of dysplastic margins, of which p53 expression may be one. Therefore, larger prospective studies are needed to confirm molecular markers for dysplastic margins. 

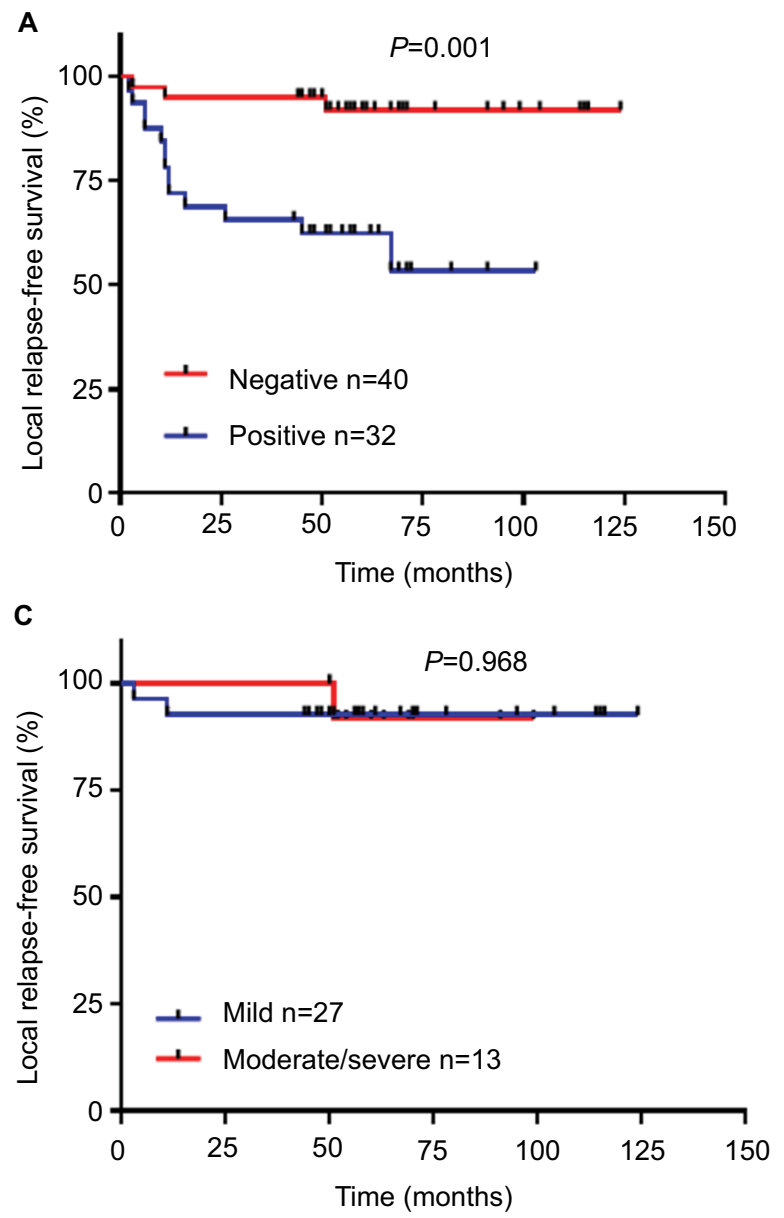

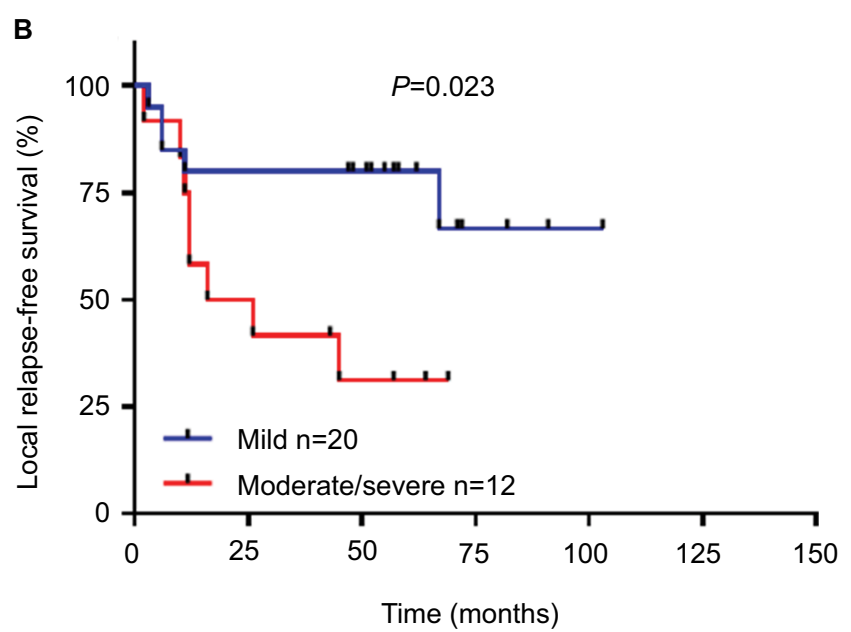

Figure 6 Grade of dysplasia and relapse-free survival (RFS) according to $p 53$ expression. Notes: (A) p53 and RFS. (B) p53-positive group. (C) p53-negative group.

Table 6 Histopathological and molecular markers that determine local relapse-free survival

\begin{tabular}{|l|l|l|l|l|}
\hline \multirow{2}{*}{ Variables } & \multicolumn{2}{|l|}{ Univariable } & \multicolumn{1}{l|}{ Multivariable } \\
\cline { 2 - 5 } & Hazard ratio (95\% Cl) & P-value & Hazard ratio (95\% Cl) & P-value \\
\hline Sex & $1.823(0.678-4.902)$ & 0.234 & & \\
Grade of dysplasia & $2.619(0.972-7.059)$ & 0.057 & & \\
Histological grade & $0.666(0.15 I-2.94 I)$ & 0.592 & & \\
Smoking history & $0.477(0.154-1.478)$ & 0.199 & & 0.06 \\
Age & $3.647(1.039-12.807)$ & $\mathbf{0 . 0 4 3}$ & $3.345(0.95 I-11.766)$ & $\mathbf{0 . 0 0 4}$ \\
P53 & $6.716(1.907-23.657)$ & $\mathbf{0 . 0 0 3}$ & $6.354(1.804-22.383)$ & \\
\hline
\end{tabular}

Note: Statistically significant values are shown in bold.

\section{Conclusion}

p53 expression and the grade of dysplasia were significantly associated with tumor recurrence for early OSCC with dysplastic surgical margins. Both p53-positive expression in dysplastic surgical margins and the grade of dysplastic margins were correlated with RFS. We further confirmed that the grade of dysplastic margins in patients with p53-positive margins was correlated with RFS, but not in those with p53-negative margins. Overall, p53 overexpres- sion was an independent risk factor for early OSCC with dysplastic surgical margins.

\section{Acknowledgments}

This study was supported by Jiangsu Province's Key Provincial Youth Talents Program (grant number QNRC2016841); Nanjing Municipal Key Medical Laboratory Constructional Project Funding (since 2012); Center of Nanjing Clinical Medicine Tumor (since 2014). 


\section{Disclosure}

The authors report no conflicts of interest in this work.

\section{References}

1. Binahmed A, Nason RW, Abdoh AA. The clinical significance of the positive surgical margin in oral cancer. Oral Oncol. 2007;43(8):780-784.

2. Iseli TA, Lin MJ, Tsui A, Guiney A, Wiesenfeld D, Iseli CE. Are wider surgical margins needed for early oral tongue cancer? J Laryngol Otol. 2012;126(3):289-294.

3. Priya SR. D'Cruz AK, Pai PS. Cut margins and disease control in oral cancers. J Cancer Res Ther. 2012;8:74-79.

4. Leemans CR, Tiwari R, Nauta JJ, van der Waal I, Snow GB. Recurrence at the primary site in head and neck cancer and the significance of neck lymph node metastases as a prognostic factor. Cancer. 1994;73(1):187-190.

5. Kurita H, Nakanishi Y, Nishizawa R, et al. Impact of different surgical margin conditions on local recurrence of oral squamous cell carcinoma. Oral Oncol. 2010;46(11):814-817.

6. Brennan JA, Mao L, Hruban RH, et al. Molecular assessment of histopathological staging in squamous-cell carcinoma of the head and neck. N Engl J Med. 1995;332(7):429-435.

7. Barnes L, Eveson JW, Reichart P, Sidransky D. World Health Organization Classification of tumours: pathology and genetics of tumours of the head and neck. Lyon: IARC Press; 2005:163-175.

8. Braakhuis BJ, Tabor MP, Leemans CR, van der Waal I, Snow GB, Brakenhoff RH. Second primary tumors and field cancerization in oral and oropharyngeal cancer: molecular techniques provide new insights and definitions. Head Neck. 2002;24(2):198-206.

9. Cruz IB, Snijders PJ, Meijer CJ, et al. p53 expression above the basal cell layer in oral mucosa is an early event of malignant transformation and has predictive value for developing oral squamous cell carcinoma. J Pathol. 1998;184(4):360-368.

10. Kurita $H$, Nakanishi $Y$, Nishizawa $R$, et al. Impact of different surgical margin conditions on local recurrence of oral squamous cell carcinoma. Oral Oncol. 2010;46(11):814-817.

11. Loree TR, Strong EW. Significance of positive margins in oral cavity squamous carcinoma. Am J Surg. 1990;160(4):410-414.

12. Sopka DM, Li T, Lango MN, et al. Dysplasia at the margin? Investigating the case for subsequent therapy in "low-risk" squamous cell carcinoma of the oral tongue. Oral Oncol. 2013;49(11):1083-1087.

13. Weijers M, Snow GB, Bezemer PD, van der Wal JE, van der Waal I. The clinical relevance of epithelial dysplasia in the surgical margins of tongue and floor of mouth squamous cell carcinoma: an analysis of 37 patients. J Oral Pathol Med. 2002;31(1):11-15.
14. Pu Y, Wang Y, Huang X, et al. The influence of mild dysplasia at the surgical margin on the prognosis of oral squamous cell carcinoma. Int J Oral Maxillofac Surg. 2016;45(11):1372-1377.

15. Mao L, Clark D. Molecular margin of surgical resections--where do we go from here? Cancer. 2015;121(12):1914-1916.

16. Wang X, Chen S, Chen X, Zhang C, Liang X. Tumor-related markers in histologically normal margins correlate with locally recurrent oral squamous cell carcinoma: a retrospective study. J Oral Pathol Med. 2016;45(2):83-88.

17. Huang X, Pateromichelakis S, Hills A, et al. p53 mutations in deep tissues are more strongly associated with recurrence than mutationpositive mucosal margins. Clin Cancer Res. 2007;13(20):6099-6106.

18. Singh J, Jayaraj R, Baxi S, et al. Immunohistochemical expression levels of p53 and eIF4E markers in histologically negative surgical margins, and their association with the clinical outcome of patients with head and neck squamous cell carcinoma. Mol Clin Oncol. 2016;4(2):166-172.

19. Jalali MM, Heidarzadeh A, Zavarei MJ, Sarmast H. p53 overexpression impacts on the prognosis of laryngeal squamous cell carcinomas. Asian Pac J Cancer Prev. 2011;12(7):1731-1734.

20. Partridge M, Li SR, Pateromichelakis S. Detection of minimal residual cancer to investigate why oral tumors recur despite seemingly adequate treatment. Clin Cancer Res. 2000;6(7):2718-2725.

21. van Houten VM, Leemans CR, Kummer JA, et al. Molecular diagnosis of surgical margins and local recurrence in head and neck cancer patients: a prospective study. Clin Cancer Res. 2004;10(11):3614-3620.

22. Tabor MP, Brakenhoff RH, Ruijter-Schippers HJ, Kummer JA, Leemans CR, Braakhuis BJ. Genetically altered fields as origin of locally recurrent head and neck cancer: a retrospective study. Clin Cancer Res. 2004;10(11):3607-3613.

23. van Houten VM, Tabor MP, van den Brekel MW, et al. Mutated p53 as a molecular marker for the diagnosis of head and neck cancer. J Pathol. 2002;198(4):476-486.

24. Hintz BL, Kagan AR, Nussbaum H, Rao AR, Chan PY, Miles J. A 'watchful waiting' policy for in situ carcinoma of the vocal cords. Arch Otolaryngol. 1981;107(12):746-751.

25. Peták I, Schwab R, Örfi L, Kopper L, Kéri G. Integrating molecular diagnostics into anticancer drug discovery. Nat Rev Drug Discov. 2010;9(7):523-535.
Cancer Management and Research

\section{Publish your work in this journal}

Cancer Management and Research is an international, peer-reviewed open access journal focusing on cancer research and the optimal use of preventative and integrated treatment interventions to achieve improved outcomes, enhanced survival and quality of life for the cancer patient. The manuscript management system is completely online and includes

\section{Dovepress}

a very quick and fair peer-review system, which is all easy to use. Visit http://www.dovepress.com/testimonials.php to read real quotes from published authors. 